\title{
Transmit Power Allocation for a Downlink Two-User Interference Channel
}

\author{
Taewon Park, Jiho Jang, Oh-Soon Shin, and Kwang Bok Lee, Senior Member, IEEE
}

\begin{abstract}
We develop the optimal transmit power allocation scheme that maximizes the total throughput for a downlink twouser interference channel. The derived optimal scheme allocates the total power to one user in better channel state, as in the greedy scheme, when the degree of interference between users exceeds a certain threshold. When it is less than the threshold, on the contrary, the transmit power is divided into two users, as in the water-filling scheme. Numerical results are presented to verify the optimality of the derived scheme and to show throughput gains over the greedy and water-filling schemes.
\end{abstract}

Index Terms-Greedy power allocation, interference channel, transmit power allocation, water-filling.

\section{INTRODUCTION}

$\mathbf{T}$ RANSMIT power allocation combined with rate adaptation is an effective means for increasing the throughput of wireless communication systems [1], [2]. The water-filling power allocation scheme has been found to be optimal in the sense of maximizing the throughput, when different data signals are transmitted through orthogonal channels [1]. On the other hand, when different data signals can fully interfere with one another, it has been found to be optimal to assign the total power to only one data signal associated with the best channel condition, which is called greedy power allocation [2]. In the case of the fully interfering channel, transmit power allocation problem has also been addressed for an uncoordinated system, where each user has an individual power constraint [3]. However, optimal power allocation problem has not been addressed for the case where multiple data signals can cause partial interference to one another as in code-division multiple-access (CDMA) systems [4]. Such a general case can be modeled as a form of interference channel [5].

In this letter, we develop the optimal transmit power allocation scheme that maximizes the total throughput for a two-user interference channel. We introduce a parameter called the portion of interference to stand for the interference channel. The derived optimal scheme is shown to change according to the portion of interference. Whenever the portion

Manuscript received July 7, 2004. The associate editor for coordinating the review process of this letter and approving it for publication was Dr. S. K. Wilson. This work was supported in part by the National Research Laboratory (NRL) Program and Brain Korea 21 Project.

T. Park and J. Jang were with the School of Electrical Engineering and Computer Science (EECS), Seoul National University (SNU), Seoul 151-742, Korea. They are now with Samsung Electronics, Korea (e-mail: (taewon.park;jiho.jang)@samsung.com).

O.-S. Shin was with the School of EECS, SNU, Seoul 151-742, Korea. $\mathrm{He}$ is now with Division of Engineering and Applied Sciences, Harvard University, MA 02138, USA (e-mail: osshin@deas.harvard.edu).

K. B. Lee is with the School of EECS, SNU, Seoul 151-742, Korea (e-mail: klee@snu.ac.kr).

Digital Object Identifier 10.1109/LCOMM.2005.01019.

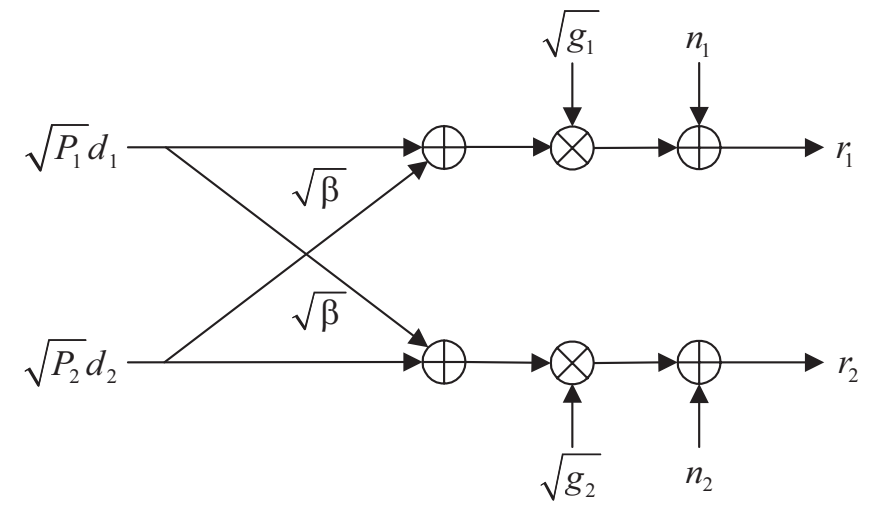

Fig. 1. Downlink two-user interference channel model.

of interference exceeds a certain threshold, the greedy scheme is found to be optimal. When it is less than the threshold, on the contrary, the transmit power is generally divided into two users, as in the water-filling scheme.

\section{Interference Channel Model And ACHIEVABLE THROUghPUT}

The interference channel considered in this paper is constructed from a downlink transmission. The base station transmits different data to two different receiving users at the same time. The data $d_{k}$ destined for the user $k$ is transmitted with power $P_{k}, k=1,2$. Assuming that the two data signals partially interfere with each other in a symmetric form, as illustrated in Fig. 1, we can express the received signal at each user as

$$
\begin{aligned}
& r_{1}=\sqrt{g_{1} P_{1}} d_{1}+\sqrt{g_{1} P_{2} \beta} d_{2}+n_{1} \\
& r_{2}=\sqrt{g_{2} P_{2}} d_{2}+\sqrt{g_{2} P_{1} \beta} d_{1}+n_{2}
\end{aligned}
$$

where $g_{k}$ denotes the channel gain between the base station and the user $k$, and $n_{1}$ and $n_{2}$ represent independent zeromean additive white Gaussian noise with variance of $\sigma^{2}$. In Fig. $1, \beta(0 \leq \beta \leq 1)$ represents the portion of interference, which quantifies the degree of interference that each data signal causes to the unintended user. The transmit powers assigned to the two users are assumed to be constrained as

$$
P_{1}+P_{2}=P \text {. }
$$

Assuming that quadrature amplitude modulation (QAM) is employed, the achievable throughput of the user $k$ normalized by the transmission bandwidth can be expressed as [2]

$$
R_{k}=\log _{2}\left(1+\gamma_{k} / \Gamma\right)
$$


where $\Gamma \equiv-\ln \left(5 P_{E}\right) / 1.5$ is the signal-to-interference-plusnoise ratio (SINR) gap, and $P_{E}$ denotes the target bit error rate (BER) required at each user. In (4), the received SINR $\gamma_{k}$ at each user can be obtained from (1) and (2) as

$$
\gamma_{1}=\frac{g_{1} P_{1}}{g_{1} P_{2} \beta+\sigma^{2}}, \gamma_{2}=\frac{g_{2} P_{2}}{g_{2} P_{1} \beta+\sigma^{2}} .
$$

From (4) and (5), the total achievable throughput normalized by the bandwidth is expressed as

$$
\begin{aligned}
R & =R_{1}+R_{2} \\
& =\log _{2}\left(\left(1+\frac{g_{1} P_{1}}{\left(g_{1} P_{2} \beta+\sigma^{2}\right) \Gamma}\right)\left(1+\frac{g_{2} P_{2}}{\left(g_{2} P_{1} \beta+\sigma^{2}\right) \Gamma}\right)\right) .
\end{aligned}
$$

\section{Optimal Transmit Power Allocation}

The optimal transmit power allocation $\left(P_{1}^{*}, P_{2}^{*}\right)$ should maximize $R$ in (6) under the constraint (3). Since the logarithm is monotonically increasing, the objective function that incorporates the power constraint can be defined as

$$
\begin{aligned}
J\left(P_{1}\right)= & \left(1+\frac{g_{1} P_{1}}{\left(g_{1}\left(P-P_{1}\right) \beta+\sigma^{2}\right) \Gamma}\right)\left(1+\frac{g_{2}\left(P-P_{1}\right)}{\left(g_{2} P_{1} \beta+\sigma^{2}\right) \Gamma}\right), \\
& 0 \leq P_{1} \leq P .
\end{aligned}
$$

Unfortunately, $J\left(P_{1}\right)$ is not guaranteed to be concave with respect to $P_{1}$. Thus, in order to find the optimal power $\left(P_{1}^{*}, P_{2}^{*}=P-P_{1}^{*}\right)$, we should search over all possible boundary points $\left(P_{1}=0\right.$ and $\left.P_{1}=P\right)$ and extreme points $\left(P_{1}\right.$ 's corresponding to $\left.\partial J / \partial P_{1}=0\right)$. First, $J\left(P_{1}\right)$ at the boundary points are computed as

$$
J\left(P_{1}=0\right)=1+\frac{g_{2} P}{\sigma^{2} \Gamma}, J\left(P_{1}=P\right)=1+\frac{g_{1} P}{\sigma^{2} \Gamma} .
$$

Next, it can easily be shown that

$$
\frac{\partial J}{\partial P_{1}}=\frac{A P_{1}^{2}+2 B P_{1}+C}{D}
$$

where

$$
\begin{aligned}
A & =g_{1} g_{2}\left(g_{1}-g_{2}\right) \beta \sigma^{2}(1-\beta \Gamma), \\
B & =g_{1} g_{2}\left(g_{1} P \beta+\sigma^{2}\right)\left(g_{2} P \beta^{2} \Gamma+(2 \beta \Gamma-1) \sigma^{2}\right)(10 \mathrm{~b}) \\
C & =-\left(g_{1} P \beta+\sigma^{2}\right)\left(g_{2} \Gamma \sigma^{2}\left(g_{2} P \beta+\sigma^{2}\right)\right. \\
& \left.+g_{1}\left(g_{2}^{2} P^{2} \beta^{2} \Gamma+g_{2} P(\beta \Gamma-1) \sigma^{2}-\Gamma \sigma^{2}\right)\right), \quad(10 \mathrm{c}) \\
D & =\left(\left(g_{1}\left(P-P_{1}\right) \beta+\sigma^{2}\right)\left(g_{2} P_{1} \beta+\sigma^{2}\right) \Gamma\right)^{2} .
\end{aligned}
$$

Since $D$ is always positive, $\partial J / \partial P_{1}=0$ is equivalent to $A P_{1}^{2}+2 B P_{1}+C=0$, which leads to

$$
P_{1}= \begin{cases}\left(-B \pm \sqrt{B^{2}-A C}\right) / A, & A \neq 0, \\ -C / 2 B, & A=0 .\end{cases}
$$

From (11), real-valued extreme points $P_{1}$ only within $[0, P]$ can be identified, and $J\left(P_{1}\right)$ at those points can be calculated using (7). Then, the optimal transmit power $P_{1}^{*}$ corresponds to the point where $J\left(P_{1}\right)$ is the largest among the two boundary points and those extreme points. From (3), $P_{2}^{*}$ is given as $P_{2}^{*}=P-P_{1}^{*}$. It can be shown that the optimal power allocation becomes identical to the water-filling scheme when $\beta=0$, and to the greedy scheme when $\beta=1$, respectively, which are given as

$$
P_{1}^{*}=\max \left(0, \frac{P}{2}+\frac{\left(g_{1}-g_{2}\right) \sigma^{2} \Gamma}{2 g_{1} g_{2}}\right), \text { if } \beta=0,
$$

$$
P_{1}^{*}=P \text { (if } g_{1} \geq g_{2} \text { ) or } 0 \text { (if } g_{1}<g_{2} \text { ), if } \beta=1 \text {. }
$$

To look into the behavior of the optimal power allocation for different $\beta$, we plot how the throughput of the optimal power allocation varies with $\beta$ in Fig. 2 , where the channel gains are assumed to be fixed to $g_{1}=2.5$ and $g_{2}=1.5$. In Fig. 2, the signal-to-noise ratio (SNR), defined to be $P / \sigma^{2}$, is assumed to be $10 \mathrm{~dB}$, and the target BER $P_{E}$ is set to $10^{-3}$. It is interesting to observe that the throughput does not change with $\beta$ if $\beta \geq \beta_{\mathrm{TH}}$, where the threshold $\beta_{\mathrm{TH}}=0.0611$ in Fig. 2 . This implies that the greedy scheme is optimal whenever $\beta \geq \beta_{\mathrm{TH}}$, since it is optimal at $\beta=1$. The threshold $\beta_{\mathrm{TH}}$ can be derived by noting that the greedy scheme provides larger throughput than any other power allocation when $\beta \geq$ $\beta_{\mathrm{TH}}$. Without loss of generality, we assume $g_{1} \geq g_{2}$, and then from (6) and (13), the normalized total throughput $R_{\mathrm{GR}}$ for the greedy scheme is given as

$$
R_{\mathrm{GR}}=\log _{2}\left(1+\frac{g_{1} P}{\sigma^{2} \Gamma}\right) .
$$

From (3), (6), and (14), the difference $\Delta\left(P_{1}\right) \equiv R_{\mathrm{GR}}-R$ can be expressed as a function of $P_{1}$ as

$$
\begin{aligned}
& \Delta\left(P_{1}\right)= \\
& \log _{2}\left(\frac{\left(\Gamma+g_{1} P / \sigma^{2}\right)\left(\left(P-P_{1}\right) \beta+\sigma^{2} / g_{1}\right)\left(P 1 \beta+\sigma^{2} / g_{2}\right) \Gamma}{\left(P \beta \Gamma-P_{1}(\beta \Gamma-1)+\Gamma \sigma^{2} / g_{1}\right)\left(P+P_{1}(\beta \Gamma-1)+\Gamma \sigma^{2} / g_{2}\right)}\right) .
\end{aligned}
$$

The range of $\beta$ that yields $\Delta\left(P_{1}\right) \geq 0$ for any value of $P_{1} \in$ $[0, P]$ can be calculated from (15), and the minimum $\beta$ in the range corresponds to $\beta_{\mathrm{TH}}$. It is derived in Appendix I as

$$
\beta_{\mathrm{TH}}=\max \left(0, \min \left(\beta_{1}, \beta_{2}\right)\right)
$$

where $\beta_{1}$ and $\beta_{2}$ are defined in (20) and (21), respectively. It can be verified that the $\beta_{\mathrm{TH}}$ calculated from (16) under the conditions of Fig. 2 is the same as the one observed in Fig. 2. In the case of $g_{1}<g_{2}, \beta_{\mathrm{TH}}$ is also given as (16) with the exchange of $g_{1}$ and $g_{2}$. In summary, the optimal transmit power allocation scheme can be written as

$$
\begin{aligned}
& P_{1}^{*}=\left\{\begin{array}{c}
\operatorname{argmax}_{p=0, P,\left(-B \pm \sqrt{B^{2}-A C}\right) / A(0 \leq p \leq P)} J(p), \\
\beta<\beta_{\mathrm{TH}}, \quad A \neq 0 \\
\operatorname{argmax}_{p=0, P,-C / 2 B}(0 \leq p \leq P) \\
\beta<\beta_{\mathrm{TH}}, A=0 \\
P, \quad \beta \geq \beta_{\mathrm{TH}}, g_{1} \geq g_{2} \\
0, \quad \beta \geq \beta_{\mathrm{TH}}, g_{1}<g_{2}
\end{array}\right. \\
& P_{2}^{*}=P-P_{1}^{*} .
\end{aligned}
$$

It should be noted that the transmit power allocation in (17) maximizes the throughput sum of the two users, not considering the individual throughput of each user. If certain fairness between the two users is desired, an adjustable weighting factor $w$ can be introduced in (6) as in [3], so that $R_{1}+w R_{2}$ rather than $R_{1}+R_{2}$ is maximized.

\section{NUMERICAL RESULTS}

Fig. 3 compares the throughput of the optimal power allocation scheme in (17) with those of the water-filling and greedy schemes in (12) and (13) in a Rayleigh fading channel, when SNR $=10 \mathrm{~dB}$ and $20 \mathrm{~dB}$. The throughput of each scheme is averaged over 10,000 independent realizations of $g_{1}$ and $g_{2}$. As expected, the optimal power allocation scheme is shown 


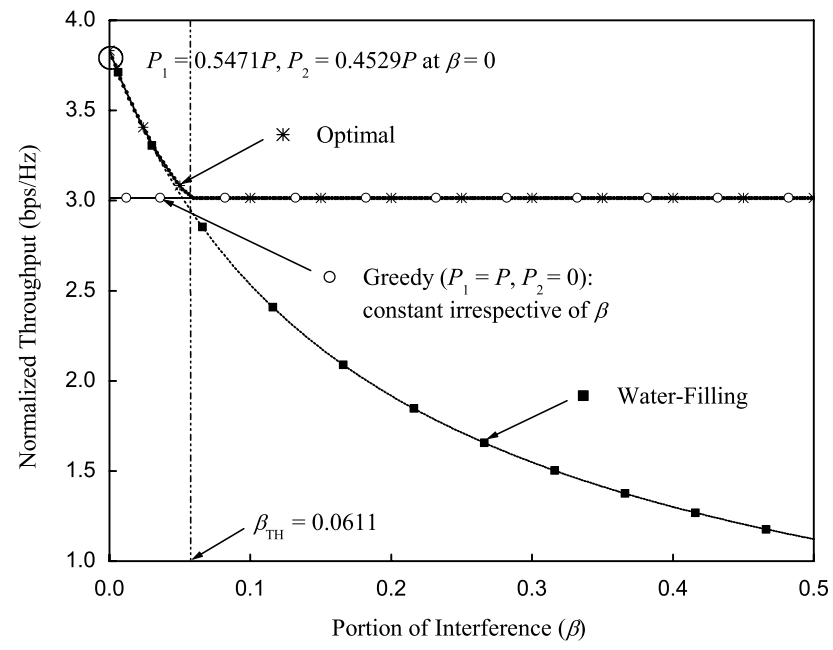

Fig. 2. Normalized throughput versus $\beta$ for given channel gains $\left(g_{1}=\right.$ $\left.2.5, g_{2}=1.5\right)$.

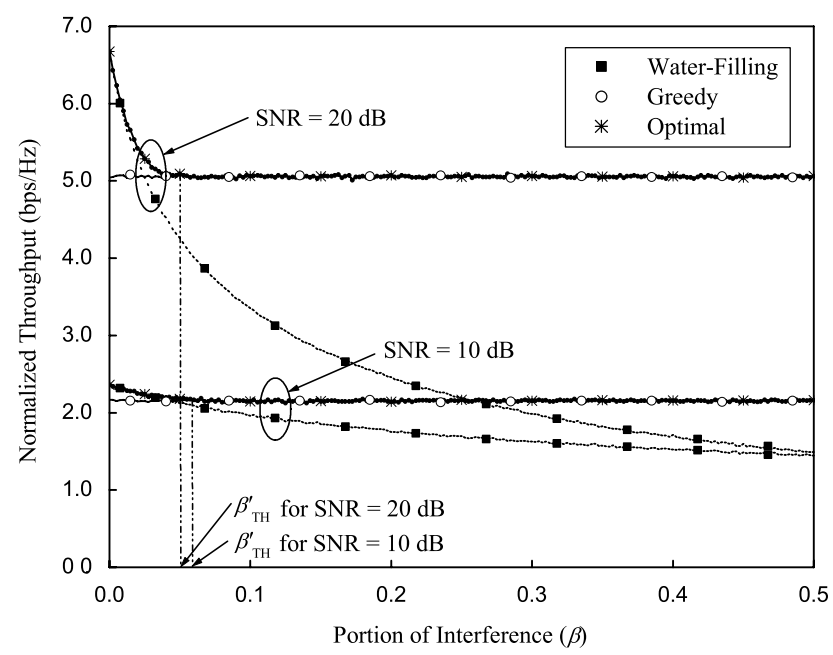

Fig. 3. Average normalized throughput versus $\beta$ in a Rayleigh fading channel.

to always achieve the maximum throughput. The throughput of the optimal scheme is shown to be indistinguishable as that of the greedy scheme when $\beta$ is greater than a certain value, denoted as $\beta_{\mathrm{TH}}^{\prime}$ in Fig. 3. This implies that $\beta_{\mathrm{TH}}$ is less than $\beta_{\mathrm{TH}}^{\prime}$ in most cases, although $\beta_{\mathrm{TH}}$ is different for different channel realizations. The throughput of the water-filling scheme is almost the same as that of the optimal scheme when $\beta<\beta_{\mathrm{TH}}^{\prime}$ , but it decreases rapidly as $\beta$ increases. When $\beta=0$ and SNR $=20 \mathrm{~dB}$, the average throughput of the optimal and water-filling schemes is $6.7 \mathrm{bps} / \mathrm{Hz}$, whereas that of the greedy scheme is $5.1 \mathrm{bps} / \mathrm{Hz}$. When $\beta=0.5$ and $\mathrm{SNR}=20 \mathrm{~dB}$, on the other hand, the optimal and greedy schemes achieve 5.1 $\mathrm{bps} / \mathrm{Hz}$, whereas the water-filling scheme achieves only 1.5 bps/Hz.

\section{Conclusions}

We have developed the optimal transmit power allocation scheme that maximizes the total throughput for a downlink two-user interference channel. The greedy scheme is found to be optimal, whenever the portion of interference $(\beta)$ is greater than a certain threshold for a given channel condition.
When $\beta$ is less than the threshold, the optimal scheme is found to behave similarly to the water-filling scheme, which is optimal at $\beta=0$. Numerical results have verified that the optimal scheme can provide significant throughput gain over the greedy scheme for relatively small $\beta$, and over the waterfilling scheme for large $\beta$.

\section{APPENDIX I}

Since the denominator of the argument of the logarithm in the right hand side of (15) is always positive, the condition $\Delta\left(P_{1}\right) \geq 0$ is equivalent to $\Omega\left(P_{1}\right) \geq 0$, where

$$
\begin{aligned}
& \Omega\left(P_{1}\right) \equiv\left(\Gamma+g_{1} P / \sigma^{2}\right)\left(\left(P-P_{1}\right) \beta+\sigma^{2} / g_{1}\right) . \\
& \left(P_{1} \beta+\sigma^{2} / g_{2}\right) \Gamma-\left(P \beta \Gamma-P_{1}(\beta \Gamma-1)+\Gamma \sigma^{2} / g_{1}\right) . \\
& \left(P+P_{1}(\beta \Gamma-1)+\Gamma \sigma^{2} / g_{2}\right) .
\end{aligned}
$$

Note that $\Omega\left(P_{1}\right)$ at the boundary points, $P_{1}=0$ and $P_{1}=P$, are given as

$$
\Omega(0)=\left(P \beta+\sigma^{2} / g_{1}\right)\left(g_{1} / g_{2}-1\right) P \Gamma \geq 0, \Omega(P)=0 .
$$

From (19), it can be seen that the range of $\beta$ that satisfies (18) for any $P_{1} \in[0, P]$ depends on the convexity of $\Omega\left(P_{1}\right)$, which can be tested from the sign of the second derivative $\partial^{2} \Omega / \partial P_{1}^{2}=-2\left(g_{1} P \beta^{2} \Gamma / \sigma^{2}+2 \beta \Gamma-1\right)$.

When $\partial^{2} \Omega / \partial P_{1}^{2} \leq 0, \Omega\left(P_{1}\right)$ is concave with respect to $P_{1}$, and thus $\Omega\left(P_{1}\right) \geq 0$ for any $P_{1}$ due to the boundary values in (19). The range of $\beta$ that satisfies $\partial^{2} \Omega / \partial P_{1}^{2} \leq 0$ is found as

$$
\beta \geq \beta_{1} \equiv \frac{-1+\sqrt{1+g_{1} P / \sigma^{2} P}}{g_{1} P / \sigma^{2}} .
$$

When $\partial^{2} \Omega / \partial P_{1}^{2} \geq 0$, on the contrary, $\Omega\left(P_{1}\right)$ is convex with respect to $P_{1}$. For $\Omega\left(P_{1}\right) \geq 0$ to be satisfied for any $P_{1}$, the minimum point of $\Omega$ should locate at a $P_{1}$ outside $[0, P]$. From these conditions, the range of $\beta$ is found as

$$
\begin{gathered}
0 \leq \beta \leq \beta_{1} \\
\beta \geq \beta_{2} \equiv \frac{-\left(g_{1}+g_{2}\right)+\sqrt{\left(g_{1}-g_{2}\right)^{2}+4 g_{2}^{2}\left(1+g_{1} P / \sigma^{2} \Gamma\right)}}{2 P g_{1} g_{2} / \sigma^{2}} .
\end{gathered}
$$

From (20) and (21), the range of $\beta$ that satisfies (18) is found as

$$
\beta \geq \max \left(0, \min \left(\beta_{1}, \beta_{2}\right)\right)
$$

which leads to $\beta_{\mathrm{TH}}=\max \left(0, \min \left(\beta_{1}, \beta_{2}\right)\right)$.

\section{REFERENCES}

[1] A. J. Goldsmith and S.-G. Chua, "Variable-rate variable-power MQAM for fading channels," IEEE Trans. Commun., vol. 45, pp. 1218-1230, Oct. 1997.

[2] J. Jang and K. B. Lee, "Transmit power adaptation for multiuser OFDM systems," IEEE J. Select. Areas Commun., vol. 21, pp. 171-178, Feb. 2003.

[3] S. T. Chung and J. M. Cioffi, "Rate and power control in a two-user multicarrier channel with no coordination: the optimal scheme versus a suboptimal method," IEEE Trans. Commun., vol. 51, pp. 1768-1772, Nov. 2003.

[4] S. Shin, J. H. Ahn, and K. Kim, "Performance comparison of CDX- and TDX-based packet scheduling schemes in DS/CDMA downlink," IEICE Trans. Commun., vol. E85-B, pp. 1914-1920, Oct. 2002.

[5] T. M. Cover and J. A. Thomas, Elements of Information Theory. New York: John Wiley \& Sons, 1991. 